withdrawal in patients with RA in remission. In a previous post hoc analysis of the AVERT trial (NCT01142726), in which several potential factors were assessed, erosions, synovitis and bone oedema scores on MRI and HAQ-DI scores in patients with DAS28 (CRP) $<2.6$ were associated with clinical relapse 6 and 12 months after complete drug withdrawal following 12 months of blinded treatment with abatacept +MTX or abatacept or MTX alone. ${ }^{1,2}$ Knowledge of the thresholds for these measures, above which relapse is more likely to occur, may aid clinical decisions about when to withdraw treatment in patients who have achieved remission.

Objectives: To evaluate post hoc the association between different thresholds of $\mathrm{MRI}$ and $\mathrm{HAQ}-\mathrm{DI}$ scores at Month 12 and the risk of relapse at Months 18 and 24 in AVERT.

Methods: This analysis compared relapse event rates at Months 18 and 24 in patients with DAS28 (CRP) <2.6 but with differing severities of synovitis, bone oedema and erosion (assessed by MRI individual and combined scores) and HAQ-DI scores at Month 12. A relapse event was defined by the doubling of TJC28 and SJC28 and an increase in DAS28 $(\mathrm{CRP}) \geq 1.2$ relative to the Month 12 visit. Synovitis, bone oedema and erosion in the dominant hand and wrist MRI were scored using the OMERACT RAMRIS. Severity of each score was defined by cut-offs of $>3$ for synovitis, $>2$ for bone oedema, $>7$ for (weighted) combined synovitis and bone oedema, $>5$ for erosion and $>0.5$ for HAQ-DI. Univariate logistic models were conducted for comparisons and odds ratios (OR) with $95 \% \mathrm{Cl}$ and associated $\mathrm{p}$ values.

Results: All randomised and treated patients with DAS28 (CRP) $<2.6$ at Month 12 and relapse status available at Months 18 and $24(n=155)$ were included. Among the overall study cohort at Month 12, 70 (45.2\%) patients with DAS28 $(\mathrm{CRP})<2.6$ had a higher synovitis score $(>3), 28(18.1 \%)$ had a higher bone oedema score (>2), $39(25.2 \%)$ had a higher combined score $(>7), 73(47.1 \%)$ had a higher erosion score $(>5)$ and $42(27.1 \%)$ had a higher HAQ-DI score $(>0.5)$. Relapse events at Months 18 and 24 were significantly associated with supra-threshold MRI bone oedema, erosion and combined scores and with suprathreshold HAQ-DI score at Month 12 (figure 1). There was a trend toward association of relapse at Months 18 and 24 with high synovitis score (figure 1).

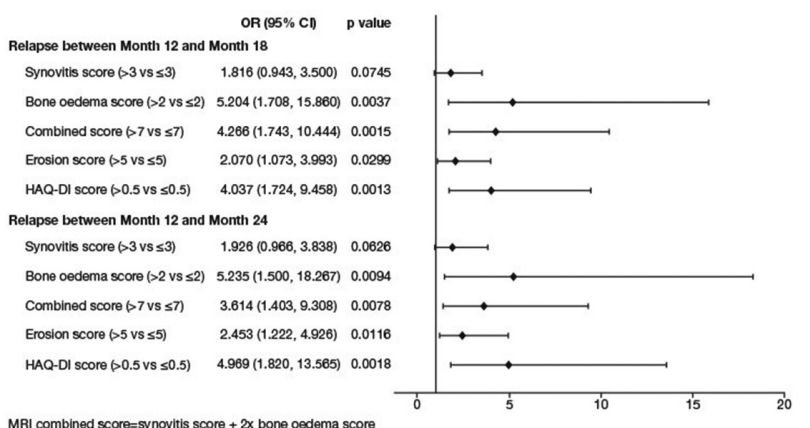

Abstract FRI0044 - Figure 1. Association Between Month 12 MRI and HAQ-DI Scores and Relapse Status at Months 18 and 24

Conclusions: It was possible to define MRI and HAQ-DI scores in patients with DAS28 (CRP) remission that were predictive of relapse 6 and 12 months after complete drug withdrawal in AVERT. Assessment of imaging and physical function, using predefined thresholds, in patients achieving remission may aid clinical decisions on when to withdraw therapy in MTX-naïve patients with RA.

\section{REFERENCES:}

[1] Emery P, et al. Ann Rheum Dis 2015;74:19-26.

[2] Ahmad H, et al. Ann Rheum Dis 2017;76(suppl 2):240.

Disclosure of Interest: H. Ahmad Shareholder of: Bristol-Myers Squibb, Employee of: Bristol-Myers Squibb, J. Baker: None declared, M. Østergaard Grant/research support from: AbbVie, Bristol-Myers Squibb, Janssen, Merck, Speakers bureau: AbbVie, Bristol-Myers Squibb, Boehringer Ingelheim, Celgene, Eli Lilly, Centocor, GSK, Hospira, Janssen, Merck, Mundipharma, Novartis, Novo Nordisk, Orion, Pfizer, Regeneron, Schering-Plough, Roche, Takeda, UCB, Wyeth, P. Emery Consultant for: AbbVie, Bristol-Myers Squibb, Merck, Pfizer, Roche, Lilly, Novartis, Samsung Bioepis, T. Huizinga Grant/research support from: EU and Dutch Arthritis Foundation, Consultant for: Abbott Laboratories, Biotest AG, Bristol-Myers Squibb, Crescendo Bioscience, Novartis, Pfizer, Roche, sanofi-aventis, Schering-Plough, UCB, Eli Lilly, Speakers bureau: Abbott Laboratories, Biotest AG, Bristol-Myers Squibb, Novartis Pharmaceuticals Corporation, Pfizer Inc, Roche, sanofi-aventis, Schering-Plough, Y. Elbez Consultant for:

Bristol-Myers Squibb, S. Banerjee Shareholder of: Bristol-Myers Squibb, Employee of: Bristol-Myers Squibb, P. Conaghan Grant/research support from: Bristol-Myers Squibb, Consultant for: AbbVie, GSK, Lilly, Novartis, Pfizer, Roche Speakers bureau: AbbVie, Bristol-Myers Squibb, Novartis DOI: 10.1136/annrheumdis-2018-eular.2117

\title{
FRI0045 EVALUATION OF SERUM PROTEIN LEVELS AT BASELINE AS PREDICTORS OF RESPONSE TO METHOTREXATE IN PATIENTS WITH EARLY RHEUMATOID ARTHRITIS: RESULTS FROM SWEFOT TRIAL POPULATION
}

K. Hambardzumyan ${ }^{1}$, C. Hamsten ${ }^{2}$, H. Idborg ${ }^{1}$, L. Lourido ${ }^{3,4}$, S. Saevarsdottir ${ }^{1}$, P. Nilsson ${ }^{3}$, R.F. van Vollenhoven ${ }^{1,5}$, P.-J. Jakobsson ${ }^{1} .{ }^{1}$ Rheumatology Unit, Department of Medicine; ${ }^{2}$ Unit of Immunology and Allergy, Department of Medicine, Karolinska Institutet, ${ }^{3}$ Affinity Proteomics, SciLifeLab, School of Biotechnology, KTH - Royal Institute of Technology, Stockholm, Sweden; ${ }^{4}$ Rheumatology Division, ProteoRed/ISCIII Proteomics Group, INIBIC - Hospital Universitario de A Coruña, A Coruña, Spain; ${ }^{5}$ Amsterdam Rheumatology and Immunology Center, Amsterdam, Netherlands

Background: Methotrexate (MTX) is a standard first-line therapy option for patients with early rheumatoid arthritis (eRA). However, a substantial proportion of patients still do not respond to MTX.

Objectives: To identify biomarkers for prediction of response to MTX.

Methods: We analysed a group of patients $(n=135)$ with eRA from the Swedish Pharmacotherapy (SWEFOT) trial population. Baseline serum levels of 177 proteins were profiled using 380 antibodies in a suspension bead array format. Median fluorescent intensity (MFI) levels of the proteins were subsequently analysed for association with achievement of low 28-joint disease activity score (DAS28 $\leq 3.2$ ) after 3 months of MTX therapy (primary outcome). Proteins that remained significant in multivariate model were analysed using receiver operating characteristic (ROC) curve analysis for cut-off definition of MFI and categorisation into high and low categories. Proportion of patients with primary outcome between the generated categories were compared using Chi-squared test.

Results: In multivariate analysis, serum levels of two of the 177 proteins at baseline, matrix metalloproteinase 7 (MMP-7) and alpha-chain of fibrinogen (FGA) were significantly different among patients who achieved or not achieved low DAS28 at 3 months. ROC curve analysis revealed AUC of 0.692 for MMP-7 and 0.699 for FGA ( $p<0.001$; figure $1 A$ ). ROC curve-based dichotomisation indicated that of patients with low versus high levels of either MMP-7 or FGA, $60 \%$ versus $24 \%$ and $58 \%$ versus $22 \%$, respectively, achieved low DAS28 $(p<0.001$ figure $1 \mathrm{~B}$ and $\mathrm{C}$ ). Among patients with low categories of both proteins, $79 \%$ achieved low DAS28 at 3 months, while only $18 \%$ of those in high categories for both proteins ( $p<0.001$; figure 1D).

Validation in the COMBINE cohort with available MMP-7 data (whose concentration was measured by different method) did not confirm results from the SWEFOT trial.

A
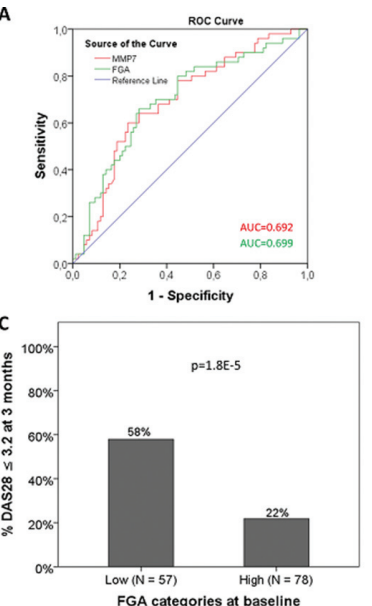

Abstract FRI0045 - Figure 1. Assessment of baseline levels of MMP-7 and FGA as predictors of LDA at 3 months. Receiver operating characteristic curve analysis and area under the curve of MMP-7 and FGA (A), proportion of patients achieving low DAS28 among groups dichotomised by MMP-7 (B), FGA (C) or using combination of MMP-7 and FGA (D). 
Conclusions: Low levels of MMP-7 and FGA at baseline were associated with better clinical outcome in eRA patients. Following further characterisation, such biomarkers would be of high clinical relevance for the optimisation of treatment of RA.

Disclosure of Interest: K. Hambardzumyan: None declared, C. Hamsten: None declared, H. Idborg: None declared, L. Lourido: None declared, S. Saevarsdottir: None declared, P. Nilsson: None declared, R. van Vollenhoven Grant/research support from: AbbVie, BMS, GSK, Pfizer, UCB, Consultant for: AbbVie, AstraZeneca, Biotest, BMS, Celgene, GSK, Janssen, Lilly, Novartis, Pfizer, UCB, P.-J. Jakobsson: None declared

DOI: 10.1136/annrheumdis-2018-eular.5408

\section{FRI0046 PATIENTS PRESENTING WITH NEW MUSCULOSKELETAL SYMPTOMS IN THE WRISTS, HANDS AND FEET ENRICHES DETECTION OF ANTI- CCP ANTIBODIES IN PRIMARY CARE - A NATIONAL COHORT STUDY}

P. Pentony $^{1,2}$, K. Karkalemis ${ }^{1}$, J.L. Nam ${ }^{1,2}$, K. Mankia ${ }^{2,3}$, L. Hunt ${ }^{1,2}$, E. M. Hensor ${ }^{1,2}$, P. Emery ${ }^{1,2}$. 'Leeds Institute of Rheumatic and Musculoskeletal Medicine, University of Leeds; ${ }^{2}$ Leeds Biomedical Research Centre, National Institute of Health Research; ${ }^{3}$ Leeds Institute of Rheumatic and Musculoskeletal Medicine, Leeds University, Leeds, UK

Background: Selecting patients with new non-specific musculoskeletal complaints can enrich the prevalence of anti-cyclic citrullinated peptide (anti-CCP) antibodies compared with the general population. ${ }^{1}$ Patients with rheumatoid arthritis (RA) frequently present with involvement of the wrist, hands and feet. Patients with elderly onset RA have a higher frequency of polymyalgic onset. However, it is unknown if elderly patients with shoulder complaints are more likely to be CCP positive.

Objectives: To confirm the proportion of individuals with new-onset, non-specific MSK symptoms who were anti-CCP positive $(\mathrm{CCP}+)$ across a national cohort and investigate the initial presenting complaint of all individuals, as this may help determine whether there is a symptom complex that would prompt antibody testing. In addition to determine if the risk of being CCP + is increased in older patients presenting with shoulder symptoms.

Methods: Individuals aged $\geq 18$ years with new musculoskeletal complaints without synovitis from primary care were recruited prospectively. Participants completed a questionnaire on baseline musculoskeletal symptoms and provided a blood sample for anti-CCP antibody (Phadia CCP-2) testing. CCP+individuals where invited to attend follow-up in the rheumatology department, Leeds. The association between CCP status, smoking and shared epitope status was also assessed.

Results: 4257 individuals were recruited, 2.9\% (125/4257) were CCP+, a significantly higher proportion compared with the general population $(1 \%(95 \% \mathrm{Cl} 2.4 \%$ $>3.5 \% \mathrm{p}<0.001)$ ). Patients who presented with pain in the wrists, hands (RR 2.2 (1.5-2.9), $\mathrm{p}<0.001$ ) or feet (RR $1.72(1.2-2.4), \mathrm{p}<0.001)$ had an increased relative risk of being $\mathrm{CCP}+$. Patients who were older than 60 years who presented with shoulder symptoms $(4.8 \%(7 / 146))$ were no more likely to be CCP +than those who did not have shoulder symptoms $(3.2 \%(31 / 1007)$, chi square $p=0.313)$ and had the same prevalence of CCP +as those $<60$ years $(3.5 \%(13 / 370)$, chi square $\mathrm{p}=0.461)$. A significantly higher proportion of ever smokers were CCP+ $(14.2 \%$ (48/337)) compared with never smokers (3.3\% (64/1926); chi square $p<0.001)$. Ever smokers were also more likely than never smokers to be shared epitope positive in CCP +individuals $(62.2 \%(23 / 37)$ cf $37.8 \%(14 / 37) ; p<0.007)$.

Conclusions: Selecting individuals with new non-specific MSK symptoms without synovitis enriched the prevalence of anti-CCP positivity to $2.9 \%$. Patients presenting with symptoms localising to the wrists, hands and feet were more likely to be CCP + which could prompt anti-CCP testing in these patients in primary care. Patients with shoulder complaints were no more likely to be CCP +than those without shoulder symptoms and had the same prevalence of CCP +as those $<60$ years.

\section{REFERENCE:}

[1] Nam JL, et al., Enriching case selection for imminent RA: the use of antiCCP antibodies in individuals with new non-specific musculoskeletal symptoms - a cohort study. Ann Rheum Dis, 2016;75(8):1452-6.

Acknowledgements: The authors would like to thank all the participating general practitioners and health professionals and the UK Clinical Research Network teams for the referrals from primary care to the study
Disclosure of Interest: None declared

DOI: 10.1136/annrheumdis-2018-eular.5368

\section{FRI0047 1 MRI INTEROSSEOUS TENDON INFLAMMATION OCCURS IN ANTI-CCP POSITIVE AT-RISK INDIVIDUALS AND MAY PRECEDE THE DEVELOPMENT OF SYNOVITIS}

K. Mankia' ${ }^{1}$, M.A. D’Agostino ${ }^{1}$, E. Hensor ${ }^{2}$, E. Rowbotham ${ }^{3}$, L. Hunt ${ }^{1}$, I. Moller ${ }^{4}$, M. Miguel ${ }^{5}$, J.L. Nam ${ }^{1}$, A.L. Tan $^{1}$, J. Freeston ${ }^{6}$, L. Garcia-Montoya ${ }^{1}$, A. Grainger ${ }^{3}$, P. Emery'. ${ }^{1}$ Rheumatology, Leeds Institute of Rheumatic and Musculoskeletal Medicine and NIHR Leeds Biomedical Research Centre; ${ }^{2}$ Rheumatology, Leeds Institute of Rheumatic and Musculoskeletal Medicine; ${ }^{3}$ Radiology, Leeds Teaching Hospitals Trust and NIHR Leeds Biomedical Research Centre, Leeds, UK; ${ }^{4}$ Rheumatology; ${ }^{5}$ Anatomy, University of Barcelona, Barcelona, Spain;

${ }^{6}$ Rheumatology, Leeds Teaching Hospitals Trust and NIHR Leeds Biomedical Research Centre, Leeds, UK

Background: Tenosynovitis (TSV) occurs in individuals at-risk of developing RA and could explain pain and stiffness in the absence of synovitis. TSV of the wrist and finger flexor tendons has been described in at-risk individuals but involvement of other hand tendons has not been well investigated. The hand interossei are crucial to hand function and can become inflamed in RA $\left(.{ }^{1}\right.$ Whether the interosseous tendons (IT) are sites of inflammation in at-risk individuals, and how this relates to joint inflammation and clinical features is unknown.

Objectives: To describe the anatomy, prevalence, pattern and clinical associations of IT inflammation in anti-CCP positive at-risk individuals.

Methods: Anti-CCP positive individuals with no synovitis (CCP+), healthy controls $(\mathrm{HC})$, DMARD-naïve early RA patients (ERA) and treated 'late' RA patients (LRA) were recruited. All subjects underwent clinical and MRI assessment. 1.5T or 3T unilateral hand MRI scans were consensus scored for RAMRIS, TSV and IT inflammation by two radiologists. IT inflammation was defined as enhancing tissue around the tendon evident in two planes. For RAMRIS and tenosynovitis, scores were adjusted for 193 age-matched controls $\left({ }^{2}\right.$ To understand the anatomical basis for MRI IT inflammation, a cadaveric study was performed on 20 fresh hand specimens; coloured dyes were injected along the first dorsal IT and into the adjacent second MCP joint and specimens were frozen and sectioned.

Results: $93 \mathrm{CCP}+, 20 \mathrm{HC}, 47 \mathrm{ERA}$ and 28 LRA were recruited. Frequency of swollen and tender joints, MRI inflammation (synovitis, BME, erosions, TSV) and CRP level increased along the RA continuum with increasing disease duration. The proportion of patients with IT inflammation increased along the RA continuum No HC, 18/93 (19\%) CCP+, 23/47 (49\%) ERA and 16/28 (57\%) LRA patients had inflammation of $>1$ IT $(p<0.001)$. The number of affected ITs increased along the RA continuum $(p<0.001)$ and tendons associated with MCPJs 2 and 5 were most commonly affected. IT inflammation and MRI synovitis were associated with MCPJ swelling [OR $2.7(0.9,8.1)$ and OR $3.1(1.0,9.8)$ respectively] but IT inflammation was the only feature independently associated with MCPJ tenderness [OR $3.1(1.4,6.8) \mathrm{p}=0.004]$. In CCP+, 99/372 (27\%) MCPJs had only one MRI abnormality; in $68 \%$ of these the abnormality was extra-capsular (57\% TSV and $11 \%$ IT inflammation). No IT sheath was identified in the cadaveric specimens suggesting the MRI findings represent peri-tendonitis rather than TSV. Dye studies indicated no clear communication between the IT and the adjacent joint (figure 1).

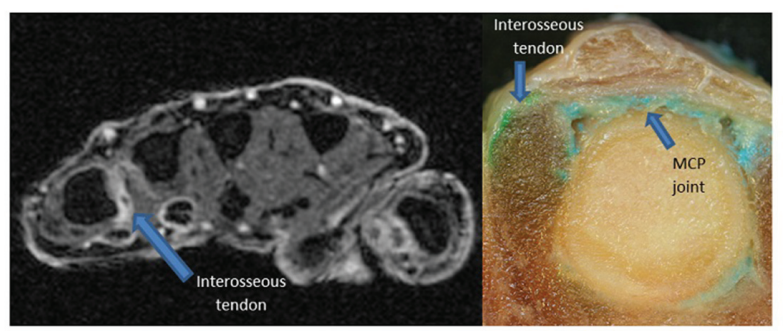

xial MRI showing inflammation of the $3^{\text {rd }}$ palmar interosseous tendon and frozen section showing green dye around the interosseous tendon and blue dye within the MCP joint

Abstract FRI0047 - Figure 1. Axial MRI showing inflammation of the 3rd palmar interosseous tendon and frozen section showing green dye around the interosseous tendon and blue dye within the MCP joint 